\title{
Capricious suntime
}

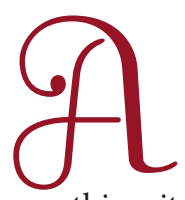

t what time of the day does the sun reach its highest point, or culmination point, when its position is exactly in the South? The answer to this question is not so trivial. For one thing, it depends on our location within our time zone. For Berlin, which is near the Eastern end of the Central European time zone, it may happen around noon, whereas in Paris it may be close to 1 p.m. (we ignore the daylight saving time which adds an extra hour in the summer). But even for a fixed location, the time at which the sun reaches its culmination point varies throughout the year in a surprising way. In other words: a sundial, however accurately positioned, will show capricious deviations through the seasons: the solar time on the

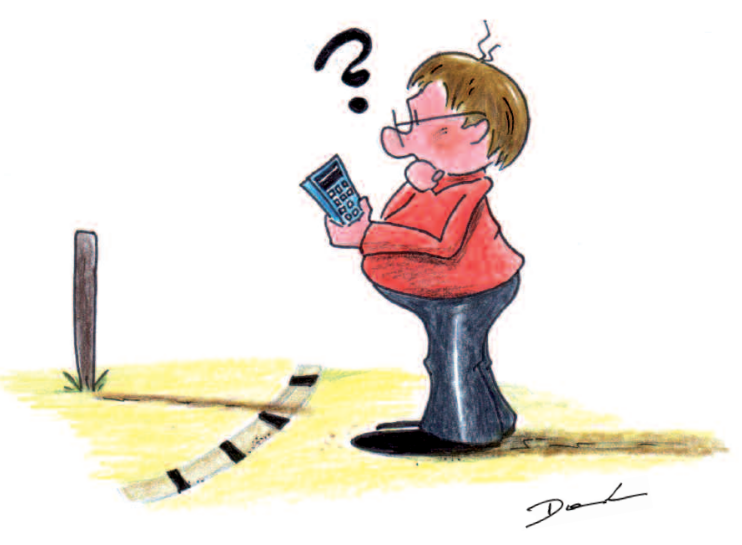

is that the solar time will gradually deviate from the time on our watch. We expect this 'eccentricity effect' to show a sine-like behaviour with a period of a year.

There is a second, even more important complication. It is due to the fact that the rotational axis of the earth is not perpendicular to the ecliptic, but is tilted by about 23.5 degrees. This is, after all, the cause of our seasons. To understand this 'tilt effect' we must realise that what matters for the deviation in time is the variation of the sun's horizontal motion against the stellar background during the year. In midsummer and mid-winter, when the sun reaches its highest and lowest point of the year, respectively, the solar motion is fully horizontal, so its effect on time is large. By contrast, in spring and autumn, the sun's path also has a vertical component, which is irrelevant here. But it makes the horizontal component smaller in these parts of the year, and so its effect on time. This gives rise to a sine-like deviation having a period of half a year.

The two contributions are shown in the graph. Superposition of these 'single and double frequency' curves yields the total deviation of the 'solar noon' from the 'mean solar noon' on our watch. We see that around February 11 the sun is about 15 minutes later than average, and around November 3 about 15 minutes earlier.

So, a sundial in our front yard may be quite charming, but understanding its readings requires a scientist. .

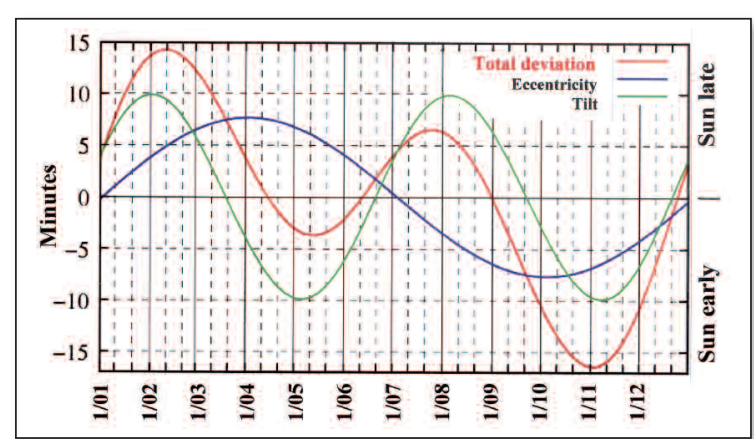

